\title{
Lentivirus-mediated short hairpin RNA targeting the APRIL gene suppresses the growth of pancreatic cancer cells in vitro and in vivo
}

\author{
FENG WANG $^{1 *}$, LIN CHEN $^{2 *}$, ZHEN-BIAO MAO ${ }^{1}$, \\ JIAN-GUO SHAO ${ }^{2}$, CHANG TAN $^{3}$ and WEI-DA HUANG ${ }^{3}$
}

${ }^{1}$ Department of Clinical Laboratory, Affiliated Hospital of Nantong University, Nantong, Jiangsu 226001;

${ }^{2}$ Department of Digestive Medicine, The Third People's Hospital of Nantong City, Nantong, Jiangsu 226006;

${ }^{3}$ Department of Biochemistry, School of Life Science, Fudan University, Shanghai 200433, P.R. China

Received January 28, 2008; Accepted April 4, 2008

\begin{abstract}
RNA interference (RNAi) is an evolutionarily conserved process of gene silencing in multiple organisms, which has become a powerful tool for investigating gene function by reverse genetics. Herein, we constructed a short hairpin RNA (shRNA) lentiviral expression vector targeting a proliferation-inducing ligand (APRIL) gene in the CFPAC-1 cell (a type of cell strain of human pancreatic cancer) in order to observe the inhibitory effect of APRIL gene's shRNA on the growth of the CFPAC-1 cell in vitro and in vivo. The results showed that lentivirus-mediated RNAi effectively inhibited the expression of APRIL mRNA and protein in CFPAC- 1 cells. Moreover, it can inhibit the growth of pancreatic cancer cells in vitro and in vivo. Our study indicates that lentivirus-mediated gene therapy is an attractive strategy in the treatment of pancreatic cancer and justifies the use of lentivirus in cancer gene therapy studies.
\end{abstract}

\section{Introduction}

Cancer cells are characterized by self-sufficiency in growth signals, insensitivity to anti-growth signals, sustained angiogenesis, tissue invasion and metastasis, limitless replicative potential and evasion of apoptosis (1). Despite the advances in surgery, radiotherapy and chemotherapy, the long-term survival rates of pancreatic cancer have not significantly improved. Gene therapy for pancreatic cancer is currently under investigation in clinical trials (2-4).

Correspondence to: Dr Jian-Guo Shao, Department of Digestive Medicine, The Third People's Hospital of Nantong City, Nantong, Jiangsu Province 226006, P.R. China

E-mail: orange303@tom.com

\section{${ }^{*}$ Contributed equally}

Key words: a proliferation-inducing ligand gene, short hairpin RNA, RNA interference, lentivirus, pancreatic cancer
RNA interference (RNAi) has been widely used in the study of gene therapy for cancer (5-7). Many groups have reported the use of synthesized oligonucleotides or siRNA encoding plasmids to induce RNAi in mammalian cells by transfection, though this is still limited in its application, especially when it is necessary to generate long-term gene silencing in vivo $(8,9)$. To circumvent this problem, lentivirusdelivered RNAi was developed. Viral vectors combined with RNAi provide useful tools in elucidating gene function by the analysis of loss-of-function phenotype and to explore the application of RNAi in gene therapy. Furthermore, the use of retroviral vectors can greatly expand the cell types available for RNAi analysis. Thus, the lentivirus vector is considered to be a promising gene delivery tool (10-12).

A proliferation-inducing ligand (APRIL) gene is a recently found new member of the tumor necrosis factor (TNF)'s superfamily. Studies have confirmed that the APRIL gene is overexpressed in many tumor tissues and tumor cell lines, particularly in the digestive system carcinomas, such as pancreatic cancer, gastric cancer, colon carcinoma, hepatoma and esophageal carcinoma etc, which suggests that APRIL plays an important role in the occurrence and development of these tumors (13-15).

Our previous experiments have further confirmed that the APRIL protein is overexpressed in pancreatic cancer tumors, yet, it is not expressed or has a weak expression in normal pancreatic gland tissues. Moreover, several pancreatic cancer cell lines have been screened and it has been found that the CFPAC-1 cell line is highly expressed in the APRIL gene. To study the effect mechanisms of the APRIL gene in the occurrence and development of pancreatic cancer, we constructed lentiviral vectors of RNAi targeting the human APRIL gene, then observed the inhibitory effect of APRIL gene's short hairpin RNA (shRNA) on the growth of CFPAC-1 cells in vitro and in vivo, in order to probe the feasibility of gene therapy for pancreatic cancer.

\section{Materials and methods}

Cell culture. The $293 \mathrm{~T}$ packaging cell line and human pancreatic cancer cell line CFPAC-1 (Academy of Life 
Science, China) were maintained in Dulbecco's modified Eagle's medium (DMEM, Invitrogen, USA), supplemented with $10 \%$ fetal bovine serum (FBS, Hyclone, Logan, USA) and antibiotics (100 mg/ml streptomycin and $100 \mathrm{U} / \mathrm{ml}$ penicillin) at $37^{\circ} \mathrm{C}$ in a humidified incubator containing $5 \% \mathrm{CO}_{2}$.

Construction of lentiviral vector. Three self-complementary hairpin DNA oligos targeting APRIL mRNA were synthesized; the sequences were 5'-TAATCCAGGATGCTG GAGTTTATTCAAGAGATAAACTCCAGCATCCTGGA TTTTTTTTC-3' and 5'-TCGAGAAAAAAAATCCAGGAT GCTGGAGTTTATCTCTTGAATAAACTCCAGCATCCT GGATTA-3', named as APRILshRNA1, 5-TAAGCCTTAT CCTACGTCCTTCTTCAAGAGAGAAGGACGTAGGAT AAGGCTTTTTTTTC-3' and 5'-TCGAGAAAAAAAAGC CTTATCCTACGTCCTTCTCTCTTGAAGAAGGACGTA GGATAAGGCTTA-3', named as APRILshRNA2, 5'-TCAT CCAGAACAGCACCACCATTTCAAGAGAATGGTGGT GCTGTTCTGGATGTTTTTTC-3' and 5'-TCGAGAAAAA ACATCCAGAACAGCACCACCATTCTCTTGAAATGGT GGTGCTGTTCTGGATGA-3', named as APRILshRNA3, including 9-nt loop sequences (underlined). A negative control to DNA oligos targeting APRIL mRNA was also designed; the sequences were 5'-TAATTCTCCGAACGTGT CACGTTTCAAGAGAACGTGACACGTTCGGAGAATT TTTTTT-3' and 5'-TCGAGAAAAAAAATTCTCCGAAC GTGTCACGTTCTCTTGAAACGTGACACGTTCGGAGA ATTA-3', named as APRILshRNA control. DNA oligos were annealed and inserted in the human U6 promoter site of the pGCL-GFP vector (Qiagen, The Netherlands). Then, the RNAi cassette was cloned into the latter vector and lentiviral vectors expressing shRNA were constructed. They were then confirmed by RT-PCR and DNA sequencing identification (3730xl DNA Analyzer, ABI, USA). The two primers targeting both sides of the pGCL-GFP vector's insert location were designed, forward primer, 5'-GTGTCACTAGGCGGG AACAC-3'; reverse primer, 5'-TTATTCCCATGCGACGGT ATC-3'.

Construction of recombinant retrovirus and transfection into CFPAC-1 cells. Lentiviral vector DNAs and packaging vectors (pHelper1.0, pHelper2.0) (Qiagen) were then transfected into $293 \mathrm{~T}$ cells. After transfection, the cells were incubated at $32^{\circ} \mathrm{C}$ to increase viral titer. Forty-eight hours later, the supernatant containing the retroviral particles was collected, filtered through the $0.45 \mu \mathrm{m}$ low protein binding syringe filter and the titer of lentiviruses was determined. CFPAC-1 cells were maintained in DMEM and were plated into 6 -well plates at $3 \times 10^{5}$ cells/well. Twenty-four hours later, the cells were infected with viral supernatants in the presence of polybrene $(6 \mu \mathrm{g} / \mathrm{ml}$ final concentration) for $12 \mathrm{~h}$ at a multiplicity of infection (MOI) of 5, then added to a fresh medium with fresh viral supernatants. After $24 \mathrm{~h}$, the cells were incubated with a fresh viral supernatant for an additional 12 h. The transfected CFPAC-1 cells were subcultured at an appropriate density in fresh DMEM and $90 \%$ of the cells were transfected at 6 days post-transfection as indicated by the expression of GFP.

Real-time RT-PCR analysis. Total RNA was extracted using an RNA purification kit from Invitrogen following the manufacturer's instructions. The real-time RT-PCR reactions were run on a real-time PCR system (iQ5 real-time PCR, Bio-Rad, USA) with the following cycle conditions: $95^{\circ} \mathrm{C}$ for $10 \mathrm{sec}, 40$ cycles at $95^{\circ} \mathrm{C}$ for $5 \mathrm{sec}$ and at $60^{\circ} \mathrm{C}$ for $30 \mathrm{sec}$. A standard curve for APRIL was created using serially diluted total RNA from CFPAC-1 cells and used to quantify relative APRIL mRNA levels. The sequences of the APRIL gene primers were: forward primer, 5'-GGTATCCCTGGCAGA GTC-3'; reverse primer, 5'-CTGTCACATCGGAGTCATC-3'; the product was $174 \mathrm{bp}$. B-actin was served as a control for normalization, the primers were: forward primer, 5'-GTGGA CATCCGCAAAGAC-3'; reverse primer, 5'-AAAGGGTGT AACGCAACTA-3'; the product was $302 \mathrm{bp}$.

Western blot analysis. The CFPAC-1 cells were lysed with a denaturing SDS-PAGE sample buffer using standard methods. Protein lysates were separated by $10 \%$ SDS-PAGE and transferred onto nitrocellulose membranes. The membranes were blocked with TBS containing $0.1 \%$ Triton X-100 and 5\% nonfat milk overnight at $4{ }^{\circ} \mathrm{C}$, then incubated with anti-human APRIL antibody (dilution at 1:1000, Prosci, CA) and B-actin (dilution at 1:5000, Santa Cruz, USA) at $4^{\circ} \mathrm{C}$ overnight. After washing, the membranes were incubated with HRP-conjugated mouse Ig at room temperature for $1 \mathrm{~h}$. Signal detection was carried out with an ECL system (Amersham Biosciences, USA).

Flow cytometry analysis of apoptosis. The CFPAC-1 cells were harvested and fixed with ice-cold $70 \%$ ethanol. Fixed samples were centrifuged at $250 \mathrm{x}$ g for $5 \mathrm{~min}$, treated with RNase $(0.25 \mathrm{mg} / \mathrm{ml})$ and re-suspended in propidium iodide $(50 \mathrm{~g} / \mathrm{ml})$ at room temperature. Propidium iodide-stained cells were analyzed by flow cytometer (Coulter Epics XL, Beckman, USA).

MTT assay cell growth viability. The cell growth following transfection was evaluated by MTT assay. Cells at a concentration of $5 \times 10^{3}$ per well were seeded in a 96 -well plate and incubated for $24 \mathrm{~h}$. Seventy-two hours after transfection, the cells were incubated with $0.5 \mathrm{mg} / \mathrm{ml}$ MTT (Sigma Chemical Co.). After $4 \mathrm{~h}$, the medium was replaced with $100 \mu 1$ dimethyl sulfoxide (DMSO) (Sigma Chemical Co.) and vortexed for $10 \mathrm{~min}$. Absorbance (A) was then recorded at $570 \mathrm{~nm}$ using an Elx800 Reader (Bio-Tek, USA). Cell viability was calculated as a percentage of the non-transfected control. All experiments were performed five times independently.

Tumorigenicity experiments and the treatment of established tumors. To determine whether APRILshRNA silence APRIL gene could inhibit tumor development in vivo, non-transfected control cells, APRILshRNA control, APRILshRNA1, APRILshRNA2 and APRILshRNA3 transfected CFPAC-1 cells $\left(1 \times 10^{6}\right.$ cells in $\left.100 \mu 1\right)$ were injected subcutaneously into the axilla of each BALB/c nude mouse. The tumor growth was monitored weekly in two dimensions and tumor size was calculated according to the formula $v=a^{2} b / 2$, where $a$ and $b$ are the shortest and longest diameters, respectively. To determine the effect of APRIL inhibition on established tumors, CFPAC- 1 cells $\left(1 \times 10^{6}\right.$ cells in $\left.100 \mu 1\right)$ were injected subcutaneously into the axilla of each mouse and allowed to develop measurable tumors. Then, the mice were treated with 


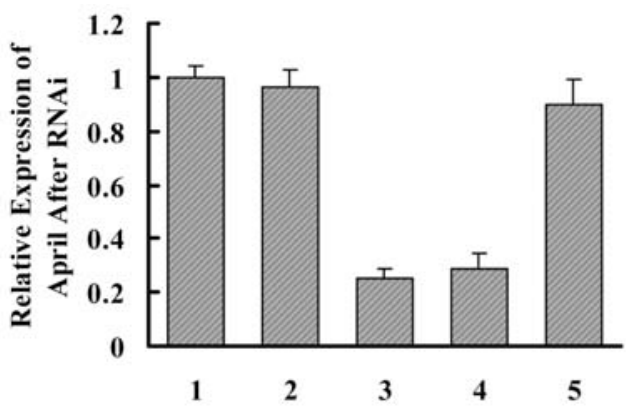

Figure 1. Expression of APRIL mRNA after CFPAC-1 cells transfected by i) non-transfected control, ii) APRILshRNA control, iii) APRILshRNA1, iv) APRILshRNA2 and v) APRILshRNA3. The image is from four independent experiments, which illustrates that APRILshRNA1 and APRILshRNA2, rather than the non-transfected control, APRILshRNA control or APRILshRNA3, reduces APRIL mRNA in CFPAC-1 cells $(\mathrm{P}<0.05)$.

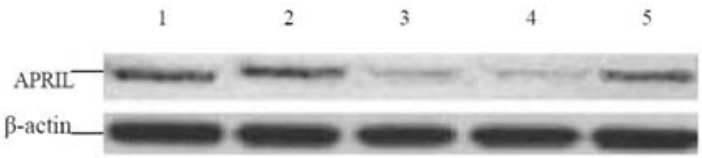

Figure 2. Expression of APRIL protein after CFPAC-1 cells transfected by i) non-transfected control, ii) APRILshRNA control, iii) APRILshRNA1, iv) APRILshRNA2 and v) APRILshRNA3. The image is from four independent experiments, which illustrates that APRILshRNA1 and APRILshRNA2, rather than the non-transfected control, APRILshRNA control or APRILshRNA3, reduces APRIL protein in CFPAC-1 cells $(\mathrm{P}<0.05)$.

the APRILshRNA control, APRILshRNA1, APRILshRNA2 and APRILshRNA3 ( $1 \times 10^{7}$ transducing units/tumor) every 5 days; the mice treated with $0.9 \%$ sodium chloride were used as controls.

Statistical analysis. Average values were expressed as mean \pm standard deviation (SD). Statistical significance between the different groups was determined by the Student's t-test. $\mathrm{P}$-values $<0.05$ were considered significant.

\section{Results}

Lentivirus-mediated RNAi inhibits APRIL gene expression in CFPAC-1 cells. In order to exclude an off-target silencing effect mediated by specific shRNA, we employed 3 different sequences of shAPRIL in our present study. To evaluate the inhibition of APRIL mRNA expression, real-time RT-PCR was performed $72 \mathrm{~h}$ after transfection, the APRIL mRNA expression in APRILshRNA1 and APRILshRNA2 transfected CFPAC-1 cells were reduced by 75 and $71 \%$, respectively, as compared with the non-transfected control and APRILshRNA control transfected ones $(\mathrm{P}<0.05)$. In addition, no difference was observed among the non-transfected control cells and the APRILshRNA control, APRILshRNA3 transfected cells (Fig. 1), indicating that the corresponding mRNA sequences for APRILshRNA1 and APRILshRNA2, though not APRIL shRNA3, are specific RNAi targets.

Lentivirus-mediated RNAi inhibits APRIL protein expression in CFPAC-1 cells. Western blot analysis was performed $72 \mathrm{~h}$ after transfection. The APRIL protein expression demonstrated

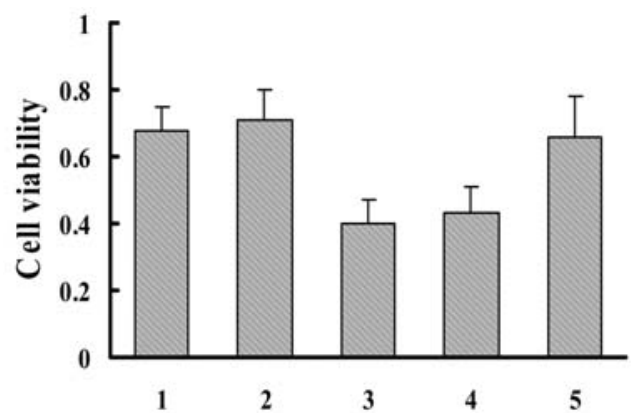

Figure 3. APRILshRNA1 and APRILshRNA2 significantly reduced cell viability assayed by MTT in CFPAC-1 cells as compared with the nontransfected control, APRILshRNA control and APRILshRNA3 transfected ones $(\mathrm{P}<0.05)$. i) Non-transfected control, ii) APRILshRNA control, iii) APRILshRNA1, iv) APRILshRNA2 and v) APRILshRNA3.

a significant reduction in APRILshRNA1 $(3.83 \pm 1.4 \%$ of $\beta$-actin) and APRILshRNA2 (4.27 $\pm 1.9 \%$ of $\beta$-actin) transfected CFPAC-1 cells respectively, as compared with a nontransfected control $(23.5 \pm 3.2 \%$ of $B$-actin), APRILshRNA control $(22.1 \pm 3.5 \%$ of $\beta$-actin $)$ and APRILshRNA3 $(19.3 \pm 4.0 \%$ of $B$-actin) transfected ones $(\mathrm{P}<0.05)$ (Fig. 2), suggesting that APRILshRNA1 and APRILshRNA2 strongly blocked APRIL expression, whereas no obvious inhibition of APRIL protein was observed in non-transfected control cells and APRILshRNA control and APRILshRNA3 transfected cells.

APRIL RNAi enhanced apoptosis and growth inhibition in CFPAC-1 cells. To further evaluate whether silencing the APRIL gene in CFPAC-1 cells may promote cell apoptosis, flow cytometry analysis was performed and the apoptosis index was calculated. In the cells transfected with the nontransfected control, APRILshRNA control, APRILshRNA1, APRIL shRNA2 and APRILshRNA3, the apoptosis index was $(4.8 \pm 0.5) \%,(4.0 \pm 0.8) \%,(15.3 \pm 0.7) \%,(14.1 \pm 1.2) \%$ and $(5.5 \pm 0.9) \%$, respectively. Apoptosis indexes were significantly higher in APRILshRNA1 and APRILshRNA2 transfected cells as compared with the non-transfected control, APRIL shRNA control and APRILshRNA3 transfected ones $(\mathrm{P}<0.05)$.

MTT analysis revealed a cell growth inhibition consistent with the results of an apoptosis analysis. As shown in Fig. 3, cell growth was significantly inhibited in APRILshRNA1 and APRILshRNA2 transfected cells as compared with the non-transfected control, APRILshRNA control and APRIL shRNA3 transfected ones $(\mathrm{P}<0.05)$.

APRIL RNAi inhibits tumor growth in vivo. To determine whether APRILshRNA silences the APRIL gene could inhibit tumor development in vivo, non-transfected control cells, APRILshRNA control, APRILshRNA1, APRILshRNA2 and APRILshRNA3 infected CFPAC-1 cells were injected into the nude mice and the tumor growth was monitored weekly. When compared with non-transfected control cells, APRILshRNA control and APRILshRNA3 infected CFPAC-1 cells, the APRIL shRNA1 and APRILshRNA2 transfected cells developed much smaller tumors in the nude mice $(\mathrm{P}<0.05)$, (Fig. 4A). 

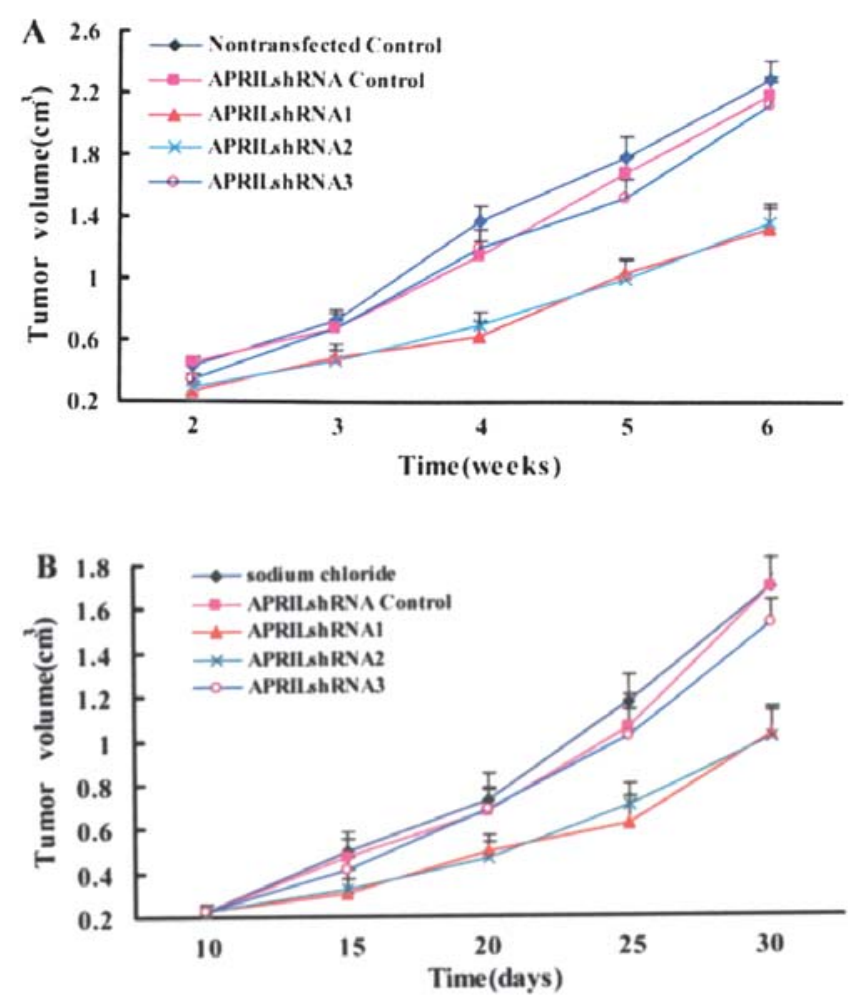

Figure 4. APRIL RNAi inhibits tumor growth in vivo. (A) Non-transfected control cells, APRILshRNA control, APRILshRNA1, APRILshRNA2 and APRILshRNA3 transfected cell growth in nude mice. APRILshRNA1 and APRILshRNA2 compared with non-transfected control, APRILshRNA control and APRILshRNA3 3 at the fourth, fifth and sixth weeks, $p<0.05$. (B) Intra-tumor injection of sodium chloride, APRILshRNA control, APRILshRNA1, APRILshRNA2 and APRILshRNA3 to observe the growth of pre-established tumors in nude mice. APRILshRNA1 and APRILshRNA2 compared with sodium chloride, APRILshRNA control and APRILshRNA3 after 25 and 30 days of injection $(\mathrm{P}<0.05)$.

Moreover, to determine the effect of APRILshRNA inhibition on established tumors, CFPAC- 1 cells were injected into the mice and were allowed to develop measurable tumors. Then the mice were treated with $0.9 \%$ sodium chloride, APRIL shRNA control, APRILshRNA1, APRILshRNA2 and APRIL shRNA3 every five days. There was no animal death in the course of the treatment and no other complications such as skin necrosis were detected due to infection. Intra-tumor injection with APRILshRNA1 and APRILshRNA2 could significantly suppress the growth of tumors, compared with being injected $0.9 \%$ sodium chloride, APRILshRNA control and APRILshRNA3 (P<0.05), (Fig. 4B).

\section{Discussion}

The phylogenetically conserved cellular phenomenon of RNAi, the sequence-specific posttranscriptional silencing of gene expression mediated by small double-stranded RNAsholds substantial promise for basic research and drug development. Particularly attractive from a medical standpoint, is the juxtaposition of new RNAi methodology with established gene transfer strategies, especially viral vectors for efficient and tissue-specific RNAi delivery to patients (16). RNAi, combined with the versatility and robustness of lentiviral vector-mediated gene delivery to a wide range of cell types offers the possibility of a long-term downregulation of specific target genes in vitro and in vivo. The use of silencing lentivectors allows for a rapid and convenient way of establishing cell lines (or transgenic mice) that stably express shRNAs for analysis of the effects produced by the knockdown of a specific gene (17).

Pancreatic cancer is the fourth leading cause of cancerrelated death in the USA. The disease has a high mortality rate and the 5-year survival rate is estimated to be $4 \%$. Currently, surgical resection is only possible in $20 \%$ of patients; even then, the overall 5-year survival rate is only 25\% (18). Despite scientific efforts and significant progress in understanding the basic cellular event in pancreatic cancer, survival rates have not changed much during the last 20 years. Prognosis in pancreatic cancer remains unsatisfactory due to its late clinical presentation, low surgical resectability rates and resistance to chemotherapy. Novel therapeutic strategies are needed urgently in order to improve the prognosis of patients with pancreatic cancer (19). During the course of cancer development, gene abnormality such as mutation or amplification can be detected frequently, which may be related with tumor survival, progression and metastasis. In human pancreatic cancer, a series of specific gene (oncogene and tumor suppressor gene) mutations are observed, including the APRIL gene.

APRIL, also known as TALL-2 and TNFSF13, is a new found mumber in the TNF superfamily. It is closely related to the B cell activation factor from the TNF family (BAFF). These two TNF ligands share the receptors, B cell maturation antigen (BCMA) and transmembrane activator, calcium modulator and cyclophilin ligand interactor (TACI) (20). Recently, APRIL binding to the heparan sulfate sidechains of proteoglycans (HSPG) was demonstrated $(21,22)$. In animal models, APRIL shows a tumor-promoting activity, as their overexpression induces the development of B cell neoplasia $(23,24)$. A direct binding onto tumor cells via HSPG was observed in vitro experiments and confirmed in situ and indicates a potential role for HSPG/APRIL interactions in the development of tumors (25).

APRIL tumor-promoting activity is not restricted to B cell lymphomas. Furthermore, APRIL was shown to provide a proliferative/survival signal to tumor cells. This activity has been observed significantly in vivo by the overexpression of APRIL in tumor cells or by blocking endogenous APRIL (26-28).

In this study, we constructed the shRNA lentiviral expression vector targeting APRIL gene in CFPAC-1 cells, then observed an inhibitory effect of APRIL gene's shRNA on the growth of CFPAC- 1 cells in vitro and in vivo. The results showed that APRILshRNA1 and APRILshRNA2, rather than the non-transfected control, APRILshRNA control or APRILshRNA3, effectively inhibited the expression of APRIL mRNA and protein in CFPAC-1 cells. Moreover, APRILshRNA1 and APRILshRNA2, rather than a non-transfected control, APRILshRNA control or APRIL shRNA3, inhibited the growth of the pancreatic cancer cells in vitro and in vivo. Our present study suggests that APRIL is a feasible RNAi target gene for pancreatic cancer. Thus, 
stable lentivirus-mediated APRILshRNA holds great promise as a novel approach for APRIL-positive pancreatic cancer treatment.

Furthermore, our study indicates that lentivirus-mediated gene therapy is an attractive strategy in the treatment of pancreatic cancer and justifies the use of lentivirus in cancer gene therapy studies. However, to further promote the technique into a gene therapeutic approach, an effective and safe protocol should be developed. In summary, further studies should focus on the lentivirus vector delivery strategies that can carry APRILshRNA specifically into APRIL-positive cancer cells with low toxicity and high efficiency.

\section{Acknowledgements}

This study was supported by the Social Development Foundation of Jiangsu Province, Grant No. BS2005029.

\section{References}

1. Hanahan D and Weinberg RA: The hallmarks of cancer. Cell 100: 57-70, 2000.

2. Cascinu S, Verdecchia L, Valeri N, Berardi R and Scartozzi M: New target therapies in advanced pancreatic cancer. Ann Oncol 5: V148-V152, 2006.

3. Sarkar FH, Banerjee S and Li Y: Pancreatic cancer: pathogenesis, prevention and treatment. Toxicol Appl Pharmacol 224: 326-336, 2007.

4. Vernejoul F, Ghenassia L, Souque A, Lulka H, Drocourt D, Cordelier P, Pradayrol L, Pyronnet S, Buscail L and Tiraby G: Gene therapy based on gemcitabine chemosensitization suppresses pancreatic tumor growth. Mol Ther 14: 758-767, 2006.

5. Sun L, Cai L, Yu Y, Meng Q, Cheng X, Zhao Y, Sui G and Zhang F: Knockdown of S-phase kinase-associated protein-2 expression in MCF-7 inhibits cell growth and enhances the cytotoxic effects of epirubicin. Acta Biochim Biophys Sin 39: 999-1007, 2007.

6. Hao JH, Gu QL, Liu BY, Li JF, Chen XH, Ji YB, Zhu ZG and Lin YZ: Inhibition of the proliferation of human gastric cancer cells SGC-7901 in vitro and in vivo using Bcl-2 siRNA. Chin Med J 120: 2105-2111, 2007.

7. Bakalova R: RNA interference-about the reality to be exploited in cancer therapy. Methods Find Exp Clin Pharmacol 29: 417-421, 2007.

8. Jazag A, Ijichi H, Kanai F, Imamura T, Guleng B, Ohta M, Imamura J, Tanaka Y, Tateishi K, Ikenoue T, Kawakami T, Arakawa Y, Miyagishi M, Taira K, Kawabe T and Omata M: Smad4 silencing in pancreatic cancer cell lines using stable RNA interference and gene expression profiles induced by transforming growth factor-beta. Oncogene 24: 662-671, 2005.

9. Imamura T, Kanai F, Kawakami T, Amarsanaa J, Ijichi H, Hoshida Y, Tanaka Y, Ikenoue T, Tateishi K, Kawabe T, Arakawa Y, Miyagishi M, Taira K, Yokosuka O and Omata M: Proteomic analysis of the TGF-beta signaling pathway in pancreatic carcinoma cells using stable RNA interference to silence Smad4 expression. Biochem Biophys Res Commun 318: 289-296, 2004.

10. Delenda C: Lentiviral vectors: Optimization of packaging, transduction and gene expression. J Gene Med 11: S125-S138, 2004.

11. Stewart SA, Dykxhoorn DM, Palliser D, Mizuno H, Yu EY, An DS, Sabatini DM, Chen IS, Hahn WC, Sharp PA, Weinberg RA and Novina CD: Lentivirus-delivered stable gene silencing by RNAi in primary cells. RNA 9: 493-501, 2003.
12. Matta H, Hozayev B, Tomar R, Chugh P and Chaudhary PM: Use of lentiviral vectors for delivery of small interfering RNA. Cancer Biol Ther 2: 206-210, 2003.

13. Hahne M, Kataoka T, Schroter M, Hofmann K, Irmler M, Bodmer JL, Schneider P, Bornand T, Holler N, French LE, Sordat B, Rimoldi D and Tschopp J: APRIL, a new ligand of the tumor necrosis factor family, stimulates tumor cell growth. J Exp Med 188: 1185-1190, 1998.

14. Roth W, Wagenknecht B, Klumpp A, Naumann U, Hahne M, Tschopp J and Weller M: APRIL, a new member of the tumor necrosis factor family, modulates death ligand-induced apoptosis. Cell Death Differ 8: 403-410, 2001.

15. Kelly K, Manos E, Jensen G, Nadauld L and Jones DA: APRIL/TRDL-1, a tumor necrosis factor-like ligand, stimulates cell death. Cancer Res 60: 1021-1027, 2000.

16. Grimm D and Kay MA: RNAi and gene therapy: A mutual attraction. Hematology Am Soc Hematol Educ Program 2007: 473-481, 2007.

17. Tiscornia G, Singer O and Verma IM: Design and cloning of lentiviral vectors expressing small interfering RNAs. Nat Protoc 1: 234-240, 2006.

18. Ducreux M, Boige V, Goere D, Deutsch E, Ezra P, Elias D and Malka D: Pancreatic cancer: from pathogenesis to cure. Best Pract Res Clin Gastroenterol 21: 997-1014, 2007.

19. Talar-Wojnarowska R and Malecka-Panas E: Molecular pathogenesis of pancreatic adenocarcinoma: potential clinical implications. Med Sci Monit 12: 186-193, 2006.

20. Kalled SL, Ambrose C and Hsu YM: The biochemistry and biology of BAFF, APRIL and their receptors. Curr Dir Autoimmun 8: 206-242, 2005.

21. Ingold K, Zumsteg A, Tardivel A, Huard B, Steiner QG, Cachero TG, Qiang F, Gorelik L, Kalled SL, Acha-Orbea H, Rennert PD, Tschopp J and Schneider P: Identification of proteoglycans as the APRIL-specific binding partners. J Exp Med 201: 1375-1383, 2005.

22. Hendriks J, Planelles L, de Jong-Odding J, Hardenberg G, Pals ST, Hahne M, Spaargaren M and Medema JP: Heparan sulfate proteoglycan binding promotes APRIL-induced tumor cell proliferation. Cell Death Differ 12: 637-648, 2005.

23. Planelles L, Carvalho-Pinto CE, Hardenberg G, Smaniotto S, Savino W, Gomez-Caro R, Alvarez-Mon M, de Jong J, Eldering E, Martinez AC, Medema J P and Hahne M: APRIL promotes B-1 cell-associated neoplasm. Cancer Cell 6: 399-408, 2004.

24. Batten M, Fletcher C, Ng LG, Groom J, Wheway J, Laabi Y, Xin X, Schneider P, Tschopp J, Mackay CR and Mackay F: TNF deficiency fails to protect BAFF transgenic mice against autoimmunity and reveals a predisposition to B cell lymphoma. J Immunol 172: 812-822, 2004.

25. Mackay F, Schneider P, Rennert P and Browning J: BAFF and APRIL: a tutorial on B cell survival. Annu Rev Immunol 21: 231-264, 2003.

26. Schwaller J, Schneider P, Mhawech-Fauceglia P, McKee T, Myit S, Matthes T, Tschopp J, Donze O, Le Gal FA and Huard B: Neutrophil-derived APRIL concentrated in tumor lesions by proteoglycans correlates with human B-cell lymphoma aggressiveness. Blood 109: 331-338, 2007.

27. Rennert P, Schneider P, Cachero TG, Thompson J, Trabach L, Hertig S, Holler N, Qian F, Mullen C, Strauch K, Browning JL, Ambrose $\mathrm{C}$ and Tschopp $\mathrm{J}$ : A soluble form of $\mathrm{B}$ cell maturation antigen, a receptor for the tumor necrosis factor family member APRIL, inhibits tumor cell growth. J Exp Med 192, 1677-1684, 2000.

28. Mhawech-Fauceglia P, Kaya G, Sauter G, McKee T, Donze O, Schwaller J and Huard B: The source of APRIL up-regulation in human solid tumor lesions. J Leukoc Biol 80: 697-704, 2006. 